\title{
DIVISOR CLASS GROUPS AND GRADED CANONICAL MODULES OF MULTISECTION RINGS
}

\author{
KAZUHIKO KURANO
}

\begin{abstract}
We describe the divisor class group and the graded canonical module of the multisection ring $T\left(X ; D_{1}, \ldots, D_{s}\right)$ for a normal projective variety $X$ and Weil divisors $D_{1}, \ldots, D_{s}$ on $X$ under a mild condition. In the proof, we use the theory of Krull domain and the equivariant twisted inverse functor.
\end{abstract}

\section{$\S 1$. Introduction}

We will describe the divisor class groups and the graded canonical modules of multisection rings associated with a normal projective variety.

Suppose that $\mathbb{Z}, \mathbb{N}_{0}$, and $\mathbb{N}$ are the set of integers, nonnegative integers, and positive integers, respectively.

Let $X$ be a normal projective variety over a field $k$ with the function field $k(X)$. We always assume that $\operatorname{dim} X>0$. We denote by $C^{1}(X)$ the set of closed subvarieties of $X$ of codimension 1. For $V \in C^{1}(X)$ and $a \in k(X)^{\times}$, we define

$$
\begin{aligned}
& \operatorname{ord}_{V}(a)=\ell_{\mathcal{O}_{X, V}}\left(\mathcal{O}_{X, V} / \alpha \mathcal{O}_{X, V}\right)-\ell_{\mathcal{O}_{X, V}}\left(\mathcal{O}_{X, V} / \beta \mathcal{O}_{X, V}\right), \\
& \operatorname{div}_{X}(a)=\sum_{V \in C^{1}(X)} \operatorname{ord}_{V}(a) \cdot V \in \operatorname{Div}(X)=\bigoplus_{V \in C^{1}(X)} \mathbb{Z} \cdot V,
\end{aligned}
$$

where $\alpha$ and $\beta$ are elements in $\mathcal{O}_{X, V}$ such that $a=\alpha / \beta$, and $\ell_{\mathcal{O}_{X, V}}()$ denotes the length as an $\mathcal{O}_{X, V}$-module.

We call an element in $\operatorname{Div}(X)$ a Weil divisor on $X$. For a Weil divisor $D=\sum n_{V} V$, we say that $D$ is effective, and we write $D \geq 0$ if $n_{V} \geq 0$ for any $V \in C^{1}(X)$. For a Weil divisor $D$ on $X$, we put

$$
H^{0}\left(X, \mathcal{O}_{X}(D)\right)=\left\{a \in k(X)^{\times} \mid \operatorname{div}_{X}(a)+D \geq 0\right\} \cup\{0\} .
$$

Received December 31, 2010. Revised April 14, 2012. Accepted August 29, 2012.

First published online September 5, 2013.

2010 Mathematics Subject Classification. Primary 14C20; Secondary 13C20.

The author's work was partially supported by Japan Society for the Promotion of Science KAKENHI grant 21540050. 
Here we note that $H^{0}\left(X, \mathcal{O}_{X}(D)\right)$ is a $k$-vector subspace of $k(X)$.

Let $D_{1}, \ldots, D_{s}$ be Weil divisors on $X$. We define the multisection rings $T\left(X ; D_{1}, \ldots, D_{s}\right)$ and $R\left(X ; D_{1}, \ldots, D_{s}\right)$ associated with $D_{1}, \ldots, D_{s}$ as follows:

$$
\begin{aligned}
T\left(X ; D_{1}, \ldots, D_{s}\right) & \\
= & \bigoplus{ }_{\left(n_{1}, \ldots, n_{s}\right) \in \mathbb{N}_{0}^{s}} H^{0}\left(X, \mathcal{O}_{X}\left(\sum_{i} n_{i} D_{i}\right)\right) t_{1}^{n_{1}} \cdots t_{s}^{n_{s}} \\
\subset & k(X)\left[t_{1}, \ldots, t_{s}\right] R\left(X ; D_{1}, \ldots, D_{s}\right) \\
= & \bigoplus \bigoplus_{\left(n_{1}, \ldots, n_{s}\right) \in \mathbb{Z}^{s}} H^{0}\left(X, \mathcal{O}_{X}\left(\sum_{i} n_{i} D_{i}\right)\right) t_{1}^{n_{1}} \cdots t_{s}^{n_{s}} \\
\subset & k(X)\left[t_{1}^{ \pm 1}, \ldots, t_{s}^{ \pm 1}\right] .
\end{aligned}
$$

We want to describe the divisor class groups and the graded canonical modules of the above rings.

For a Weil divisor $F$ on $X$, we set

$$
M_{F}=\bigoplus_{\left(n_{1}, \ldots, n_{s}\right) \in \mathbb{Z}^{s}} H^{0}\left(X, \mathcal{O}_{X}\left(\sum_{i} n_{i} D_{i}+F\right)\right) t_{1}^{n_{1}} \cdots t_{s}^{n_{s}} \subset k(X)\left[t_{1}^{ \pm 1}, \ldots, t_{s}^{ \pm 1}\right]
$$

that is, $M_{F}$ is a $\mathbb{Z}^{s}$-graded reflexive $R\left(X ; D_{1}, \ldots, D_{s}\right)$-module with

$$
\left[M_{F}\right]_{\left(n_{1}, \ldots, n_{s}\right)}=H^{0}\left(X, \mathcal{O}_{X}\left(\sum_{i} n_{i} D_{i}+F\right)\right) t_{1}^{n_{1}} \cdots t_{s}^{n_{s}} .
$$

We denote by $\overline{M_{F}}$ the isomorphism class of the reflexive module $M_{F}$ in $\mathrm{Cl}\left(R\left(X ; D_{1}, \ldots, D_{s}\right)\right)$.

For a normal variety $X$, we denote by $\mathrm{Cl}(X)$ the class group of $X$, and for a Weil divisor $F$ on $X$, we denote by $\bar{F}$ the residue class represented by the Weil divisor $F$ in $\mathrm{Cl}(X)$.

In the case where $\mathrm{Cl}(X)$ is freely generated by $\overline{D_{1}}, \ldots, \overline{D_{s}}$, the ring $R\left(X ; D_{1}, \ldots, D_{s}\right)$ is usually called the Cox ring of $X$ and is denoted by $\operatorname{Cox}(X)$.

REMARK 1.1. Assume that $D$ is an ample divisor on $X$. In this case, $T(X ; D)$ coincides with $R(X ; D)$, and it is a Noetherian normal domain by a famous result of Zariski (see [6, Lemma 2.8]). It is well known that $\mathrm{Cl}(T(X ; D))$ is isomorphic to $\mathrm{Cl}(X) / \mathbb{Z} \bar{D}$. Mori in $[8]$ constructed a lot of examples of non-Cohen-Macaulay factorial domains using this isomorphism. 
It is well known that the canonical module of $T(X ; D)$ is isomorphic to $M_{K_{X}}$ and that the canonical sheaf $\omega_{X}$ coincides with $\widetilde{M_{K_{X}}}$. Watanabe proved a more general result in [12, Theorem 2.8].

We want to establish the same type of the above results for multisection rings.

For $R\left(X ; D_{1}, \ldots, D_{s}\right)$, we had already proven the following.

Theorem 1.2 ([2, Theorem 1.1], [5, Theorem 1.2]). Let $X$ be a normal projective variety over a field such that $\operatorname{dim} X>0$. Assume that $D_{1}, \ldots, D_{s}$ are Weil divisors on $X$ such that $\mathbb{Z} D_{1}+\cdots+\mathbb{Z} D_{s}$ contains an ample Cartier divisor. Then, we have the following.

(1) The ring $R\left(X ; D_{1}, \ldots, D_{s}\right)$ is a Krull domain.

(2) The set $\left\{P_{V} \mid V \in C^{1}(X)\right\}$ coincides with the set of homogeneous prime ideals of $R\left(X ; D_{1}, \ldots, D_{s}\right)$ of height 1 , where $P_{V}=M_{-V}$.

(3) We have an exact sequence

$$
0 \longrightarrow \sum_{i} \mathbb{Z} \overline{D_{i}} \longrightarrow \mathrm{Cl}(X) \stackrel{p}{\longrightarrow} \mathrm{Cl}\left(R\left(X ; D_{1}, \ldots, D_{s}\right)\right) \longrightarrow 0
$$

such that $p(\bar{F})=\overline{M_{F}}$.

(4) Assume that $R\left(X ; D_{1}, \ldots, D_{s}\right)$ is Noetherian. Then $\omega_{R\left(X ; D_{1}, \ldots, D_{s}\right)}$ is isomorphic to $M_{K_{X}}$ as a $\mathbb{Z}^{s}$-graded module. Therefore, $\omega_{R\left(X ; D_{1}, \ldots, D_{s}\right)}$ is $R\left(X ; D_{1}, \ldots, D_{s}\right)$-free if and only if $\overline{K_{X}} \in \sum_{i} \mathbb{Z} \overline{D_{i}}$ in $\mathrm{Cl}(X)$.

Suppose that $\mathrm{Cl}(X)$ is a finitely generated free $\mathbb{Z}$-module generated by $\overline{D_{1}}, \ldots, \overline{D_{s}}$. By the above theorem, the $\operatorname{Cox} \operatorname{ring} \operatorname{Cox}(X)$ is factorial, and

$$
\omega_{\operatorname{Cox}(X)}=M_{K_{X}}=\operatorname{Cox}(X)\left(\overline{K_{X}}\right)
$$

where we regard $\operatorname{Cox}(X)$ as a $\mathrm{Cl}(X)$-graded ring.

The main result of this paper is the following.

THEOREM 1.3. Let $X$ be a normal projective variety over a field $k$ such that $d=\operatorname{dim} X>0$. Assume that $D_{1}, \ldots, D_{\text {s }}$ are Weil divisors on $X$ such that $\mathbb{N} D_{1}+\cdots+\mathbb{N} D_{s}$ contains an ample Cartier divisor. Put

$$
U=\left\{j \mid \operatorname{tr} \cdot \operatorname{deg}_{k} T\left(X ; D_{1}, \ldots, D_{j-1}, D_{j+1}, \ldots, D_{s}\right)=d+s-1\right\} .
$$

Then, we have the following.

(1) The ring $T\left(X ; D_{1}, \ldots, D_{s}\right)$ is a Krull domain. 
(2) The set

$$
\left\{Q_{V} \mid V \in C^{1}(X)\right\} \cup\left\{Q_{j} \mid j \in U\right\}
$$

coincides with the set of homogeneous prime ideals of $T\left(X ; D_{1}, \ldots, D_{s}\right)$ of height 1, where

$$
Q_{V}=P_{V} \cap T\left(X ; D_{1}, \ldots, D_{s}\right)
$$

and

$$
Q_{j}=\bigoplus_{\substack{n_{1}, \ldots, n_{s} \in \mathbb{N}_{0} \\ n_{j}>0}} T\left(X ; D_{1}, \ldots, D_{s}\right)_{\left(n_{1}, \ldots, n_{s}\right)}
$$

(3) We have an exact sequence

$$
0 \longrightarrow \sum_{j \notin U} \mathbb{Z} \overline{D_{j}} \longrightarrow \mathrm{Cl}(X) \stackrel{q}{\longrightarrow} \mathrm{Cl}\left(T\left(X ; D_{1}, \ldots, D_{s}\right)\right) \longrightarrow 0
$$

such that $q(\bar{F})=\overline{M_{F} \cap k(X)\left[t_{1}, \ldots, t_{s},\left\{t_{j}^{-1} \mid j \notin U\right\}\right]}$.

(4) Assume that $T\left(X ; D_{1}, \ldots, D_{s}\right)$ is Noetherian. Then $\omega_{T\left(X ; D_{1}, \ldots, D_{s}\right)}$ is isomorphic to

$$
M_{K_{X}} \cap t_{1} \cdots t_{s} k(X)\left[t_{1}, \ldots, t_{s},\left\{t_{j}^{-1} \mid j \notin U\right\}\right]
$$

as a $\mathbb{Z}^{s}$-graded module. Further, we have

$$
q\left(\overline{K_{X}+\sum_{i} D_{i}}\right)=\overline{\omega_{T\left(X ; D_{1}, \ldots, D_{s}\right)}}
$$

Therefore, $\omega_{T\left(X ; D_{1}, \ldots, D_{s}\right)}$ is $T\left(X ; D_{1}, \ldots, D_{s}\right)$-free if and only if

$$
\overline{K_{X}+\sum_{i} D_{i}} \in \sum_{j \notin U} \mathbb{Z} \overline{D_{j}}
$$

in $\mathrm{Cl}(X)$.

Here, $\operatorname{tr} \cdot \operatorname{deg}_{k} T$ denotes the transcendence degree of the fractional field of $T$ over a field $k$.

REMARK 1.4. With notation as in Theorem $1.3, \operatorname{ht}\left(Q_{j}\right)=1$ if and only if $j \in U$. This will be proved in Lemma 3.3. Since $\mathbb{N} D_{1}+\cdots+\mathbb{N} D_{s}$ contains an ample Cartier divisor, $Q_{j} \neq(0)$ for any $j$. Therefore, $\operatorname{ht}\left(Q_{j}\right) \geq 2$ if and only if $j \notin U$. 


\section{§2. Examples}

EXAmple 2.1. Let $X$ be a normal projective variety with $\operatorname{dim} X>0$. Assume that all $D_{i}$ are ample Cartier divisors on $X$. Then, $T\left(X ; D_{1}, \ldots, D_{s}\right)$ is Noetherian by a famous result of Zariski (see [6, Lemma 2.8]).

Assume that $s=1$. By definition, $U=\emptyset$ since $\operatorname{dim} X>0$. By Theorem 1.3(3), $\mathrm{Cl}\left(T\left(X ; D_{1}\right)\right)$ is isomorphic to $\mathrm{Cl}(X) / \mathbb{Z} \overline{D_{1}}$. By Theorem 1.3(4), $\omega_{T\left(X ; D_{1}\right)}$ is a $T\left(X ; D_{1}\right)$-free module if and only if

$$
\overline{K_{X}} \in \mathbb{Z} \overline{D_{1}}
$$

in $\mathrm{Cl}(X)$ (see Remark 1.1).

Next, assume that $s \geq 2$. In this case, $U=\{1,2, \ldots, s\}$. By Theorem $1.3(3), \mathrm{Cl}(X)$ is isomorphic to $\mathrm{Cl}\left(T\left(X ; D_{1}, \ldots, D_{s}\right)\right)$. By Theorem 1.3(4), $\omega_{T\left(X ; D_{1}, \ldots, D_{s}\right)}$ is a $T\left(X ; D_{1}, \ldots, D_{s}\right)$-free module if and only if

$$
\overline{K_{X}}=\overline{-D_{1}-\cdots-D_{s}}
$$

in $\mathrm{Cl}(X)$. When this is the case, $-K_{X}$ is ample; that is, $X$ is a Fano variety.

ExAmple 2.2. Set $X=\mathbb{P}^{m} \times \mathbb{P}^{n}$. Let $p_{1}$ (resp., $p_{2}$ ) be the first (resp., second) projection.

Let $H_{1}$ be a hyperplane of $\mathbb{P}^{m}$, and let $H_{2}$ be a hyperplane of $\mathbb{P}^{n}$. Put $A_{i}=p_{i}^{-1}\left(H_{i}\right)$ for $i=1,2$. In this case, $\mathrm{Cl}(X)=\mathbb{Z} \overline{A_{1}}+\mathbb{Z} \overline{A_{2}} \simeq \mathbb{Z}^{2}$, and $K_{X}=$ $-(m+1) A_{1}-(n+1) A_{2}$.

We have

$$
\operatorname{Cox}(X)=R\left(X ; A_{1}, A_{2}\right)=k\left[x_{0}, x_{1}, \ldots, x_{m}, y_{0}, y_{1}, \ldots, y_{n}\right] .
$$

$\operatorname{Cox}(X)$ is a $\mathbb{Z}^{2}$-graded ring such that $x_{i}$ (resp., $\left.y_{j}\right)$ are of degree $(1,0)$ (resp., $(0,1))$.

Let $a, b, c, d$ be positive integers such that $a d-b c \neq 0$. Put $D_{1}=a A_{1}+$ $b A_{2}$, and put $D_{2}=c A_{1}+d A_{2}$. Then, both $D_{1}$ and $D_{2}$ are ample divisors. Consider the multisection rings

$$
\begin{aligned}
& R\left(X ; D_{1}, D_{2}\right)=\bigoplus_{p, q \in \mathbb{Z}} \operatorname{Cox}(X)_{p(a, b)+q(c, d)}, \\
& T\left(X ; D_{1}, D_{2}\right)=\bigoplus_{p, q \geq 0} \operatorname{Cox}(X)_{p(a, b)+q(c, d)} .
\end{aligned}
$$

Here, both $R\left(X ; D_{1}, D_{2}\right)$ and $T\left(X ; D_{1}, D_{2}\right)$ are Cohen-Macaulay rings. 
By Theorem 1.2(4), we know that

$$
\begin{aligned}
R\left(X ; D_{1}, D_{2}\right) \text { is a Gorenstein ring } & \Longleftrightarrow \overline{K_{X}} \in \mathbb{Z} \overline{D_{1}}+\mathbb{Z} \overline{D_{2}} \text { in } \mathrm{Cl}(X) \\
& \Longleftrightarrow(m+1, n+1) \in \mathbb{Z}(a, b)+\mathbb{Z}(c, d) .
\end{aligned}
$$

In this case, we have $U=\{1,2\}$ since all of $a, b, c$, and $d$ are positive. By Theorem 1.3(4), we have

$$
\begin{aligned}
T\left(X ; D_{1}, D_{2}\right) \text { is a Gorenstein ring } & \Longleftrightarrow \overline{K_{X}+D_{1}+D_{2}}=0 \text { in } \mathrm{Cl}(X) \\
& \Longleftrightarrow m+1=a+c \text { and } n+1=b+d .
\end{aligned}
$$

EXAMPLE 2.3. Let $a, b, c$ be pairwise coprime positive integers. Let $\mathfrak{p}$ be the kernel of the $k$-algebra map $S=k[x, y, z] \rightarrow k[T]$ given by $x \mapsto T^{a}$, $y \mapsto T^{b}, z \mapsto T^{c}$.

Let $\pi: X \rightarrow \mathbb{P}=\operatorname{Proj}(k[x, y, z])$ be the blowup at $V_{+}(\mathfrak{p})$, where $a=\operatorname{deg}(x)$, $b=\operatorname{deg}(y), c=\operatorname{deg}(z)$. Put $E=\pi^{-1}\left(V_{+}(\mathfrak{p})\right)$. Let $A$ be a Weil divisor on $X$ satisfying $\pi^{*} \mathcal{O}_{\mathbb{P}}(1)=\mathcal{O}_{X}(A)$. In this case, we have $\mathrm{Cl}(X)=\mathbb{Z} \bar{E}+\mathbb{Z} \bar{A} \simeq \mathbb{Z}^{2}$, and $K_{X}=E-(a+b+c) A$.

Then, we have

$$
\begin{aligned}
\operatorname{Cox}(X) & =R(X ;-E, A)=R_{s}^{\prime}(\mathfrak{p}):=S\left[t^{-1}, \mathfrak{p} t, \mathfrak{p}^{(2)} t^{2}, \mathfrak{p}^{(3)} t^{3}, \ldots\right] \subset S\left[t^{ \pm 1}\right], \\
T(X ;-E, A) & =R_{s}(\mathfrak{p}):=S\left[\mathfrak{p} t, \mathfrak{p}^{(2)} t^{2}, \mathfrak{p}^{(3)} t^{3}, \ldots\right] \subset S[t] .
\end{aligned}
$$

By Theorem 1.2(4), we have

$$
\omega_{R_{s}^{\prime}(\mathfrak{p})}=M_{K_{X}}=R_{s}^{\prime}(\mathfrak{p})\left(\overline{K_{X}}\right)=R_{s}^{\prime}(\mathfrak{p})(-1,-a-b-c) .
$$

In this case, $U=\{1\}$. By Theorem 1.3(4), we have

$$
\begin{aligned}
\omega_{R_{s}(\mathfrak{p})} & =M_{K_{X}} \cap t_{1} t_{2} k(X)\left[t_{1}, t_{2}^{ \pm 1}\right] \\
& =\omega_{R_{s}^{\prime}(\mathfrak{p})} \cap t_{1} t_{2} k(X)\left[t_{1}, t_{2}^{ \pm 1}\right] \\
& =R_{s}^{\prime}(\mathfrak{p})(-1,-a-b-c) \cap t_{1} t_{2} k(X)\left[t_{1}, t_{2}^{ \pm 1}\right] \\
& =R_{s}(\mathfrak{p})(-1,-a-b-c) .
\end{aligned}
$$

Therefore, both of $R_{s}^{\prime}(\mathfrak{p})$ and $R_{s}(\mathfrak{p})$ are quasi-Gorenstein rings that were first proved by Simis and Trung [11, Corollary 3.4]. The Cohen-Macaulayness of such rings are deeply studied by Goto, Nishida, and Shimoda [3].

Divisor class groups of ordinary and symbolic Rees rings were studied by, for example, Shimoda [10] and Simis and Trung [11]. 


\section{§3. Proof of Theorem 1.3}

Throughout this section, we assume that $X$ is a normal projective variety over a field $k$ such that $d=\operatorname{dim} X>0$, and we assume that $D_{1}, \ldots, D_{s}$ are Weil divisors on $X$ such that $\mathbb{N} D_{1}+\cdots+\mathbb{N} D_{s}$ contains an ample Cartier divisor.

We need the following lemmas. They are well known, but the author could not find a reference.

Lemma 3.1. Let $G$ be an integral domain containing a field $k$. Let $P$ be a prime ideal of $G$. Assume that both tr. $\operatorname{deg}_{k} G$ and $\operatorname{tr} \cdot \operatorname{deg}_{k} G / P$ are finite.

Then, the height of $P$ is less than or equal to

$$
\text { tr. } \operatorname{deg}_{k} G-\operatorname{tr} \cdot \operatorname{deg}_{k} G / P \text {. }
$$

Proof. Assume the contrary. Then there exists a ring $G^{\prime}$ which satisfies the following five conditions:

- $k \subset G^{\prime} \subset G$;

- $G^{\prime}$ is finitely generated (as a ring) over $k$;

- tr. $\operatorname{deg}_{k} G=\operatorname{tr} . \operatorname{deg}_{k} G^{\prime}$;

- tr. $\operatorname{deg}_{k} G / P=\operatorname{tr} . \operatorname{deg}_{k} G^{\prime} /\left(G^{\prime} \cap P\right)$; and

- tr. $\operatorname{deg}_{k} G-\operatorname{tr} \cdot \operatorname{deg}_{k} G / P<\operatorname{ht}\left(G^{\prime} \cap P\right)$.

However, using the dimension formula (e.g., [7, p. 119]), we have

$$
\operatorname{ht}\left(G^{\prime} \cap P\right)=\operatorname{tr} \cdot \operatorname{deg}_{k} G^{\prime}-\operatorname{tr} \cdot \operatorname{deg}_{k} G^{\prime} /\left(G^{\prime} \cap P\right)=\operatorname{tr} \cdot \operatorname{deg}_{k} G-\operatorname{tr} \cdot \operatorname{deg}_{k} G / P \text {. }
$$

This is a contradiction.

Lemma 3.2. Let $r$ be a positive integer. Let $F_{1}, \ldots, F_{r}$ be Weil divisors on $X$. Let $S$ be the set of all nonzero homogeneous elements of $T\left(X ; F_{1}, \ldots\right.$, $\left.F_{r}\right)$. Then the following conditions are equivalent.

(1) There exist nonnegative integers $q_{1}, \ldots, q_{r}$ such that $\sum_{i=1}^{r} q_{i} F_{i}$ is linearly equivalent to a sum of an ample Cartier divisor and an effective Weil divisor.

(2) There exist positive integers $q_{1}, \ldots, q_{r}$ such that $\sum_{i=1}^{r} q_{i} F_{i}$ is linearly equivalent to a sum of an ample Cartier divisor and an effective Weil divisor.

(3) We have $S^{-1}\left(T\left(X ; F_{1}, \ldots, F_{r}\right)\right)=k(X)\left[t_{1}^{ \pm 1}, \ldots, t_{r}^{ \pm 1}\right]$.

(4) We have $Q\left(T\left(X ; F_{1}, \ldots, F_{r}\right)\right)=k(X)\left(t_{1}, \ldots, t_{r}\right)$, where $Q()$ denotes the field of fractions.

(5) We have tr.deg $\operatorname{deg}_{k} T\left(X ; F_{1}, \ldots, F_{r}\right)=\operatorname{dim} X+r$. 
Using $[1$, Theorem 1.5 .5$]$, it is easy to see that $T\left(X ; F_{1}, \ldots, F_{r}\right)$ is Noetherian if and only if $T\left(X ; F_{1}, \ldots, F_{r}\right)$ is finitely generated (as a ring) over the field $H^{0}\left(X, \mathcal{O}_{X}\right)$. Therefore, if $T\left(X ; F_{1}, \ldots, F_{r}\right)$ is Noetherian, then condition (5) is equivalent to stating that the Krull dimension of $T\left(X ; F_{1}, \ldots, F_{r}\right)$ is $\operatorname{dim} X+r$.

Proof. Here $(2) \Rightarrow(1)$, and $(3) \Rightarrow(4) \Rightarrow(5)$ are trivial.

First we will prove that $(1) \Rightarrow(3)$. Suppose that

$$
\sum_{i=1}^{r} q_{i} F_{i} \sim D+F
$$

where $q_{i}$ are nonnegative integers, $D$ is a very ample Cartier divisor, and $F$ is an effective divisor. We put

$$
\begin{aligned}
C & =\bigoplus_{m \in \mathbb{Z}\left(n_{1}, \ldots, n_{r}\right) \in \mathbb{N}_{0}^{r}} H^{0}\left(X, \mathcal{O}_{X}\left(\sum_{i} n_{i} F_{i}+m D\right)\right) t_{1}^{n_{1}} \cdots t_{r}^{n_{r}} t_{r+1}^{m} \\
& \subset k(X)\left[t_{1}, \ldots, t_{r}, t_{r+1}^{ \pm 1}\right] .
\end{aligned}
$$

We regard $C$ as a $\mathbb{Z}^{r+1}$-graded ring with

$$
C_{\left(n_{1}, \ldots, n_{r}, m\right)}=H^{0}\left(X, \mathcal{O}_{X}\left(\sum_{i} n_{i} F_{i}+m D\right)\right) t_{1}^{n_{1}} \cdots t_{r}^{n_{r}} t_{r+1}^{m} .
$$

Then, we have

$$
T\left(X ; F_{1}, \ldots, F_{r}\right)=\bigoplus_{\left(n_{1}, \ldots, n_{r}\right) \in \mathbb{N}_{0}^{r}} C_{\left(n_{1}, \ldots, n_{r}, 0\right)},
$$

so $T\left(X ; F_{1}, \ldots, F_{r}\right)$ is a subring of $C$. Thus, $S^{-1} C$ is a $\mathbb{Z}^{r+1}$-graded ring such that

$$
S^{-1} T\left(X ; F_{1}, \ldots, F_{r}\right)=\bigoplus_{\left(n_{1}, \ldots, n_{r}\right) \in \mathbb{N}_{0}^{r}}\left(S^{-1} C\right)_{\left(n_{1}, \ldots, n_{r}, 0\right)} .
$$

Since $\sum_{i=1}^{r} q_{i} F_{i}-D$ is linearly equivalent to an effective divisor $F$, there exists a nonzero element $a$ in

$$
H^{0}\left(X, \mathcal{O}_{X}\left(\sum_{i} q_{i} F_{i}-D\right)\right)
$$


For any $0 \neq b \in H^{0}\left(X, \mathcal{O}_{X}(D)\right)$,

$$
\left(a t_{1}^{q_{1}} \cdots t_{r}^{q_{r}} t_{r+1}^{-1}\right)\left(b t_{r+1}\right)
$$

is contained in $S$. Therefore, $S^{-1} C$ contains $\left(b t_{r+1}\right)^{-1}$. Hence, $k(X)$ is contained in $S^{-1} C$. Since $k(X)=\left(S^{-1} C\right)_{(0, \ldots, 0)}, k(X)$ is contained in $S^{-1} T(X$; $\left.F_{1}, \ldots, F_{r}\right)$.

By assumption (1), there exists a positive integer $\ell$ such that

$$
\left(S^{-1} C\right)_{\left(\ell q_{1}, \ldots, \ell q_{r}, 0\right)} \neq 0
$$

and

$$
\left(S^{-1} C\right)_{\left(\ell q_{1}+1, \ell q_{2}, \ldots, \ell q_{r}, 0\right)} \neq 0 .
$$

Then, it is easy to see that $t_{1} \in S^{-1} C$. Therefore, $S^{-1} C$ contains $k(X)\left[t_{1}^{ \pm 1}\right.$, $\left.\ldots, t_{r}^{ \pm 1}\right]$. Hence, $S^{-1} T\left(X ; F_{1}, \ldots, F_{r}\right)$ coincides with $k(X)\left[t_{1}^{ \pm}, \ldots, t_{r}^{ \pm}\right]$.

Next, we will prove $(5) \Rightarrow(2)$. Let $D$ be a very ample divisor. Consider the ring

$$
R\left(X ; F_{1}, \ldots, F_{r}, D\right)
$$

First, assume that

$$
H^{0}\left(X, \mathcal{O}_{X}\left(\sum_{i} u_{i} F_{i}-v D\right)\right) \neq 0
$$

for some integers $u_{1}, \ldots, u_{r}, v$ such that $v>0$. By assumption (5), there exist positive integers $u_{1}^{\prime}, \ldots, u_{r}^{\prime}$ such that

$$
H^{0}\left(X, \mathcal{O}_{X}\left(\sum_{i} u_{i}^{\prime} F_{i}\right)\right) \neq 0
$$

Therefore, we may assume that there exist positive integers $u_{1}, \ldots, u_{r}$ and $v$ such that

$$
H^{0}\left(X, \mathcal{O}_{X}\left(\sum_{i} u_{i} F_{i}-v D\right)\right) \neq 0 .
$$

Here, we have

$$
\sum_{i} u_{i} F_{i}=v D+\left(\sum_{i} u_{i} F_{i}-v D\right) .
$$

Therefore, $\sum_{i} u_{i} F_{i}$ is the sum of an ample divisor $v D$ and the divisor $\sum_{i} u_{i} F_{i}-v D$, which is linearly equivalent to an effective divisor. 
Next, assume that for any integers $u_{1}, \ldots, u_{r}$ and $v$,

$$
H^{0}\left(X, \mathcal{O}_{X}\left(\sum_{i} u_{i} F_{i}-v D\right)\right)=0
$$

if $v>0$. We put

$$
P=\bigoplus_{\substack{\left(n_{1}, \ldots, n_{r}, m\right) \in \mathbb{Z}^{r+1} \\ m>0}} R\left(X ; F_{1}, \ldots, F_{r}, D\right)_{\left(n_{1}, \ldots, n_{r}, m\right)} .
$$

By assumption (5), $P$ is a prime ideal of $R\left(X ; F_{1}, \ldots, F_{r}, D\right)$ of height 1 by Lemma 3.1. (Here, since $D$ is an ample divisor, $\operatorname{tr} . \operatorname{deg}_{k} R\left(X ; F_{1}, \ldots, F_{r}, D\right)=$ $\operatorname{dim} X+r+1$. Note that $P$ is an ideal of $R\left(X ; F_{1}, \ldots, F_{r}, D\right)$ by $(3.2)$ above. By $(5), \operatorname{tr} . \operatorname{deg}_{k} R\left(X ; F_{1}, \ldots, F_{r}, D\right) / P=\operatorname{dim} X+r$.) However, $R\left(X ; F_{1}, \ldots\right.$, $\left.F_{r}, D\right)$ has no homogeneous prime ideal of height 1 that contains

$$
H^{0}\left(X, \mathcal{O}_{X}(D)\right) t_{r+1}
$$

by Theorem 1.2(2). This is a contradiction.

Put $A=k(X)\left[t_{1}^{ \pm 1}, \ldots, t_{s}^{ \pm 1}\right]$, and put $B=k(X)\left[t_{1}, \ldots, t_{s}\right]$. Recall that $D_{1}$, $\ldots, D_{s}$ are Weil divisors on a normal projective variety $X$ such that $\mathbb{N} D_{1}+$ $\cdots+\mathbb{N} D_{s}$ contains an ample Cartier divisor. We denote $T\left(X ; D_{1}, \ldots, D_{s}\right)$ and $R\left(X ; D_{1}, \ldots, D_{s}\right)$ simply by $T$ and $R$, respectively.

Since

$$
T=R \cap B,
$$

$T$ is a Krull domain. We have proved Theorem 1.3(1).

By Theorem 1.2(2), we have

$$
\begin{aligned}
& R=\left(\bigcap_{V \in C^{1}(X)} R_{P_{V}}\right) \cap A, \\
& A=\bigcap_{P \in \operatorname{NHP}^{1}(R)} R_{P},
\end{aligned}
$$

where $\operatorname{NHP}^{1}(R)$ is the set of nonhomogeneous prime ideals of $R$ of height 1 .

It is easy to see that $R_{P}=T_{P \cap T}$ for $P \in \operatorname{NHP}^{1}(R)$. Therefore, we have

$$
A=\bigcap_{P \in \mathrm{NHP}^{1}(R)} T_{P \cap T}
$$

Since $T_{P \cap T}$ is a discrete valuation ring, $P \cap T$ is a nonhomogeneous prime ideal of $T$ of height 1 . 
For $V \in C^{1}(X)$, put $Q_{V}=P_{V} \cap T$. Then, $R_{P_{V}}=T_{Q_{V}}$, since $\sum_{i} \mathbb{N} D_{i}$ contains an ample divisor. Therefore, $Q_{V}$ is a homogeneous prime ideal of $T$ of height 1 .

On the other hand, we have $Q_{i}=T \cap t_{i} B_{\left(t_{i}\right)}$ and $T_{Q_{i}} \subset B_{\left(t_{i}\right)}$. Note that

$$
B=A \cap\left(\bigcap_{j=1}^{s} B_{\left(t_{j}\right)}\right)
$$

Then, we have

$$
\begin{aligned}
T & =R \cap B \\
& =\left(\bigcap_{V \in C^{1}(X)} R_{P_{V}}\right) \cap A \cap B \\
& =\left(\bigcap_{V \in C^{1}(X)} T_{Q_{V}}\right) \cap\left(\bigcap_{P \in \operatorname{NHP}^{1}(R)} T_{P \cap T}\right) \cap\left(\bigcap_{j=1}^{s} B_{\left(t_{j}\right)}\right) .
\end{aligned}
$$

Put

$$
T_{j}=\bigoplus_{\left(n_{1}, \ldots, n_{j-1}, n_{j+1}, \ldots, n_{s}\right) \in \mathbb{N}_{0}^{s-1}} H^{0}\left(X, \mathcal{O}_{X}\left(\sum_{i \neq j} n_{i} D_{i}\right)\right) t_{1}^{n_{1}} \cdots t_{j-1}^{n_{j-1}} t_{j+1}^{n_{j+1}} \cdots t_{s}^{n_{s}} .
$$

We need the following lemma.

LEMMA 3.3. With notation as above, the following conditions are equivalent:

(1) $T_{Q_{j}}=B_{\left(t_{j}\right)}$

(2) the height of $Q_{j}$ is 1 ;

(3) the height of $Q_{j}$ is less than 2 ; and

(4) $j \in U$, that is, tr.deg $T_{j}=d+s-1$.

Proof. By Lemma 3.2, we have $Q(T)=Q(B)$. It is easy to see that $B_{\left(t_{j}\right)}$ is a discrete valuation ring. Since $Q_{j}$ is a nonzero prime ideal of a Krull domain $T$, the equivalence of (1), (2), and (3) is easy to see.

Here, we will prove $(1) \Rightarrow(4)$. Note that $T / Q_{j}=T_{j}$. Then, we have

$$
Q\left(T_{j}\right)=T_{Q_{j}} / Q_{j} T_{Q_{j}}=B_{\left(t_{i}\right)} /\left(t_{i}\right) B_{\left(t_{i}\right)}=k(X)\left(t_{1}, \ldots, t_{j-1}, t_{j+1}, \ldots, t_{s}\right) .
$$

The implication that $(4) \Rightarrow(3)$ immediately follows from

$$
\text { ht }\left(Q_{j}\right) \leq \operatorname{tr} \cdot \operatorname{deg}_{k} T-\operatorname{tr} \cdot \operatorname{deg}_{k}\left(T_{j}\right)=1 .
$$

This inequality follows from Lemma 3.1 and from the fact that $T_{j}=T / Q_{j}$. 
By (3.3), Lemma 3.3, and [7, Theorem 12.3], we know that

$$
\left\{Q_{V} \mid V \in C^{1}(X)\right\} \cup\left\{Q_{j} \mid j \in U\right\}
$$

is the set of homogeneous prime ideals of $T$ of height 1 , and that

$$
\left\{P \cap T \mid P \in \mathrm{NHP}^{1}(R)\right\}
$$

is the set of nonhomogeneous prime ideals of $T$ of height 1 . Further, we obtain

$$
T=\left(\bigcap_{V \in C^{1}(X)} T_{Q_{V}}\right) \cap\left(\bigcap_{P \in \operatorname{NHP}^{1}(R)} T_{P \cap T}\right) \cap\left(\bigcap_{j \in U} T_{Q_{j}}\right) .
$$

The proof of Theorem 1.3(2) is completed.

Let

$$
\operatorname{Div}(X)=\bigoplus_{V \in C^{1}(X)} \mathbb{Z} \cdot V
$$

be the set of Weil divisors on $X$. Let

$$
\operatorname{HDiv}(T)=\left(\bigoplus_{V \in C^{1}(X)} \mathbb{Z} \cdot \operatorname{Spec}\left(T / Q_{V}\right)\right) \oplus\left(\bigoplus_{j \in U} \mathbb{Z} \cdot \operatorname{Spec}\left(T / Q_{j}\right)\right)
$$

be the set of homogeneous Weil divisors of $\operatorname{Spec}(T)$.

Here, we define

$$
\phi: \operatorname{Div}(X) \longrightarrow \operatorname{HDiv}(T)
$$

by $\phi(V)=\operatorname{Spec}\left(T / Q_{V}\right)$ for each $V \in C^{1}(X)$. Then, it satisfies the following.

- For each $a \in k(X)^{\times}$, we have

$$
\phi\left(\operatorname{div}_{X}(a)\right)=\operatorname{div}_{T}(a) \in \bigoplus_{V \in C^{1}(X)} \mathbb{Z} \cdot \operatorname{Spec}\left(T / Q_{V}\right) \subset \operatorname{HDiv}(T) .
$$

- If $j \in U$, then

$$
\operatorname{div}_{T}\left(t_{j}\right)=\operatorname{Spec}\left(T / Q_{j}\right)+\phi\left(D_{j}\right)
$$

- If $j \notin U$, then

$$
\operatorname{div}_{T}\left(t_{j}\right)=\phi\left(D_{j}\right)
$$


They are proven essentially in the same way as in [2, pp. 631-632]. Then, we have an exact sequence

$$
0 \longrightarrow \sum_{j \notin U} \mathbb{Z} \overline{D_{j}} \longrightarrow \mathrm{Cl}(X) \stackrel{q}{\longrightarrow} \mathrm{Cl}(T) \longrightarrow 0
$$

such that $q(\bar{F})=\overline{\phi(F)}$ in $\mathrm{Cl}(T)$. Here, remember that $\mathrm{Cl}(T)$ coincides with $\operatorname{HDiv}(T)$ divided by the group of homogeneous principal divisors (see, e.g., [9, Proposition 7.1]).

It is easy to see that the class of the Weil divisor $q(\bar{F})$ corresponds to the isomorphism class of the reflexive module

$$
\begin{aligned}
M_{F} \cap\left(\bigcap_{j \in U} T_{Q_{j}}\right) & =M_{F} \cap A \cap\left(\bigcap_{j \in U} T_{Q_{j}}\right) \\
& =M_{F} \cap k(X)\left[t_{1}, \ldots, t_{s},\left\{t_{j}^{-1} \mid j \notin U\right\}\right] .
\end{aligned}
$$

The proof of Theorem 1.3(3) is completed.

REMARK 3.4. It is easy to see that

$$
t_{1}^{d_{1}} \cdots t_{s}^{d_{s}} M_{F+\sum_{i} d_{i} D_{i}}=M_{F}
$$

for any integers $d_{1}, \ldots, d_{s}$. Therefore, we have

$$
\begin{aligned}
M_{F} & \cap t_{1}^{d_{1}} \cdots t_{s}^{d_{s}} k(X)\left[t_{1}, \ldots, t_{s},\left\{t_{j}^{-1} \mid j \notin U\right\}\right] \\
\quad= & t_{1}^{d_{1}} \cdots t_{s}^{d_{s}}\left(M_{F+\sum_{i} d_{i} D_{i}} \cap k(X)\left[t_{1}, \ldots, t_{s},\left\{t_{j}^{-1} \mid j \notin U\right\}\right]\right) .
\end{aligned}
$$

Hence,

$$
M_{F} \cap t_{1}^{d_{1}} \cdots t_{s}^{d_{s}} k(X)\left[t_{1}, \ldots, t_{s},\left\{t_{j}^{-1} \mid j \notin U\right\}\right]
$$

is isomorphic to

$$
M_{F+\sum_{i} d_{i} D_{i}} \cap k(X)\left[t_{1}, \ldots, t_{s},\left\{t_{j}^{-1} \mid j \notin U\right\}\right]
$$

as a $T$-module. Note that this is not an isomorphism as a $\mathbb{Z}^{s}$-graded module. The isomorphism class to which module (3.4) belongs coincides with $q\left(\overline{F+\sum_{i} d_{i} D_{i}}\right)$.

In the rest, we assume that $T$ is Noetherian. We will prove that $\omega_{T}$ is isomorphic to

$$
M_{K_{X}} \cap t_{1} \cdots t_{s} k(X)\left[t_{1}, \ldots, t_{s},\left\{t_{j}^{-1} \mid j \notin U\right\}\right]
$$


as a $\mathbb{Z}^{s}$-graded module. (Suppose that it is true. If we forget the grading, it is isomorphic to

$$
M_{K_{X}+\sum_{i} D_{i}} \cap k(X)\left[t_{1}, \ldots, t_{s},\left\{t_{j}^{-1} \mid j \notin U\right\}\right]
$$

by Remark 3.4, that is, corresponding to $q\left(\overline{K_{X}+\sum_{i} D_{i}}\right)$ in $\mathrm{Cl}(T)$. Therefore, we know that $\omega_{T}$ is $T$-free if and only if

$$
\overline{K_{X}+\sum_{i} D_{i}} \in \sum_{j \notin U} \mathbb{Z} \overline{D_{j}}
$$

\section{in $\mathrm{Cl}(X)$.)}

Put $X^{\prime}=X \backslash \operatorname{Sing}(X)$. We choose positive integers $a_{1}, \ldots, a_{s}$ and sections $f_{1}, \ldots, f_{t} \in H^{0}\left(X, \sum_{i} a_{i} D_{i}\right)$ such that

- $\sum_{i} a_{i} D_{i}$ is an ample Cartier divisor,

- $X^{\prime}=\bigcup_{k} D_{+}\left(f_{k}\right)$, and

- all of the $D_{i}$ are principal Cartier divisors on $D_{+}\left(f_{k}\right)$ for $k=1, \ldots, t$.

Put $W=\left\{\underline{n} \in \mathbb{Z}^{s} \mid n_{i} \geq 0\right.$ if $\left.i \in U\right\}$. Put $D_{i}^{\prime}=\left.D_{i}\right|_{X^{\prime}}$ for $i=1, \ldots, s$. Consider the morphism

$$
Y=\operatorname{Spec}_{X^{\prime}}\left(\bigoplus_{\underline{n} \in W} \mathcal{O}_{X^{\prime}}\left(\sum_{i} n_{i} D_{i}^{\prime}\right) t_{1}^{n_{1}} \cdots t_{s}^{n_{s}}\right) \stackrel{\pi}{\longrightarrow} X^{\prime} .
$$

Further, we have the natural map

$$
\xi: Y \longrightarrow \operatorname{Spec}(T)
$$

The group $\mathbb{G}_{m}^{s}$ naturally acts on $\operatorname{Spec}(T)$ and $Y$ and trivially acts on $X^{\prime}$. Both $\pi$ and $\xi$ are equivariant morphisms.

Claim 3.5. There exists an equivariant open subscheme $Z$ of both $Y$ and $\operatorname{Spec}(T)$ such that

- the codimension of $Y \backslash Z$ in $Y$ is greater than or equal to 2, and

- the codimension of $\operatorname{Spec}(T) \backslash Z$ in $\operatorname{Spec}(T)$ is greater than or equal to 2 .

Proof. For $u \in U$, there exist integers $c_{1 u}, \ldots, c_{s u}$ such that

- $H^{0}\left(X, \mathcal{O}_{X}\left(\sum_{i} c_{i u} D_{i}\right)\right) \neq 0$,

- $c_{u u}=-a_{u}$, and

- $c_{i u}>0$ if $i \neq u$. 
In fact, if $u \in U$, there exist positive integers $q_{1}, \ldots, q_{u-1}, q_{u+1}, \ldots, q_{s}$ such that

$$
\sum_{i \neq u} q_{i} D_{i}
$$

is a sum of an ample divisor $D$ and a Weil divisor $F$, which is linearly equivalent to an effective divisor by Lemma 3.2. Then,

$$
H^{0}\left(X, \mathcal{O}_{X}\left(q\left(\sum_{i \neq u} q_{i} D_{i}\right)-a_{u} D_{u}\right)\right)=H^{0}\left(X, \mathcal{O}_{X}\left(q(D+F)-a_{u} D_{u}\right)\right) \neq 0
$$

for $q \gg 0$.

For each $u \in U$, we set

$$
\left(b_{1 u}, \ldots, b_{s u}\right)=\left(c_{1 u}, \ldots, c_{s u}\right)+\left(a_{1}, \ldots, a_{s}\right) .
$$

Here, note that $b_{u u}=0$ and $b_{i u}>0$ if $i \neq u$.

We choose

$$
0 \neq g_{u} \in H^{0}\left(X, \mathcal{O}_{X}\left(\sum_{i} c_{i u} D_{i}\right)\right)
$$

for each $u \in U$.

Consider the closed set of $\operatorname{Spec}(T)$ defined by the ideal $J$ generated by

$$
\left\{f_{k} t_{1}^{a_{1}} \cdots t_{s}^{a_{s}} \mid k=1, \ldots, t\right\}
$$

and

$$
\left\{g_{u} f_{k} t_{1}^{b_{1 u}} \cdots t_{s}^{b_{s u}} \mid k=1, \ldots, t ; u \in U\right\} .
$$

By Theorem 1.3(2), we know that the height of $J$ is greater than or equal to 2 since there is no prime ideal of $T$ of height 1 which contains $J$.

We choose $d_{k i} \in k(X)^{\times}$satisfying

$$
H^{0}\left(D_{+}\left(f_{k}\right), \mathcal{O}_{X}\left(D_{i}\right)\right)=d_{k i} H^{0}\left(D_{+}\left(f_{k}\right), \mathcal{O}_{X}\right)
$$

for each $k$ and $i$. Then

$$
Y=\bigcup_{k=1}^{t} \pi^{-1}\left(D_{+}\left(f_{k}\right)\right) \quad \text { and } \quad \pi^{-1}\left(D_{+}\left(f_{k}\right)\right)=\operatorname{Spec}\left(C_{k}\right),
$$

where

$$
C_{k}=H^{0}\left(D_{+}\left(f_{k}\right), \mathcal{O}_{X}\right)\left[d_{k 1} t_{1}, \ldots, d_{k s} t_{s},\left\{\left(d_{k j} t_{j}\right)^{-1} \mid j \notin U\right\}\right] .
$$

We put

$$
Z=\operatorname{Spec}(T) \backslash V(J)
$$


Then we have

$$
\begin{aligned}
Z= & \bigcup_{k=1}^{t}\left[\operatorname{Spec}\left(T\left[\left(f_{k} t_{1}^{a_{1}} \cdots t_{s}^{a_{s}}\right)^{-1}\right]\right)\right. \\
& \left.\cup\left\{\bigcup_{u \in U} \operatorname{Spec}\left(T\left[\left(g_{u} f_{k} t_{1}^{b_{1 u}} \cdots t_{s}^{b_{s u}}\right)^{-1}\right]\right)\right\}\right] .
\end{aligned}
$$

Here, we have

$$
\begin{aligned}
T\left[\left(f_{k} t_{1}^{a_{1}} \cdots t_{s}^{a_{s}}\right)^{-1}\right] & =H^{0}\left(D_{+}\left(f_{k}\right), \mathcal{O}_{X}\right)\left[\left(d_{k 1} t_{1}\right)^{ \pm 1}, \ldots,\left(d_{k s} t_{s}\right)^{ \pm 1}\right] \\
& =C_{k}\left[\left(\prod_{j \in U}\left(d_{k j} t_{j}\right)\right)^{-1}\right]
\end{aligned}
$$

On the other hand,

$$
\begin{aligned}
T & {\left[\left(g_{u} f_{k} t_{1}^{b_{1 u}} \cdots t_{s}^{b_{s u}}\right)^{-1}\right] } \\
= & \bigoplus_{(\underline{n}) \in \mathbb{Z}^{s}} T\left[\left(g_{u} f_{k} t_{1}^{b_{1 u}} \cdots t_{s}^{b_{s u}}\right)^{-1}\right]_{\left(n_{1}, \ldots, n_{s}\right)} \\
= & \bigoplus_{(\underline{n}) \in \mathbb{Z}^{s}} R\left[\left(g_{u} f_{k} t_{1}^{b_{1 u}} \cdots t_{s}^{b_{s u}}\right)^{-1}\right]_{\left(n_{1}, \ldots, n_{s}\right)} \\
= & \bigoplus_{(\underline{n}) \in 0} R\left[\left(f_{k} t_{1}^{a_{1}} \cdots t_{s}^{a_{s}}\right)^{-1},\left(g_{u} f_{k} t_{1}^{b_{1 u}} \cdots t_{s}^{b_{s u}}\right)^{-1}\right]_{\left(n_{1}, \ldots, n_{s}\right)} \\
= & C_{k}\left[\left\{\left(d_{k j} t_{j}\right)^{-1} \mid j \neq u\right\},\left(g_{u} f_{k} t_{1}^{b_{1 u}} \cdots t_{s}^{b_{s u}}\right)^{-1}\right] .
\end{aligned}
$$

Let $\beta_{k u}$ be an element in $H^{0}\left(D_{+}\left(f_{k}\right), \mathcal{O}_{X}\right)$ such that

$$
g_{u} f_{k} t_{1}^{b_{1 u}} \cdots t_{s}^{b_{s u}}=\beta_{k u}\left(d_{k 1} t_{1}\right)^{b_{1 u}} \cdots\left(d_{k s} t_{s}\right)^{b_{s u}}
$$

for $k=1, \ldots, t$ and $u \in U$. Then,

$$
\begin{aligned}
C_{k} & {\left[\left\{\left(d_{k j} t_{j}\right)^{-1} \mid j \neq u\right\},\left(g_{u} f_{k} t_{1}^{b_{1 u}} \cdots t_{s}^{b_{s u}}\right)^{-1}\right] } \\
& =C_{k}\left[\left(\beta_{k u} \prod_{\substack{j \in U \\
j \neq u}}\left(d_{k j} t_{j}\right)\right)^{-1}\right] .
\end{aligned}
$$


By (3.5), (3.6), (3.7), and (3.8), we know that $Z$ is an open subscheme of $Y$. The ideal of $C_{k}$ generated by

$$
\prod_{j \in U}\left(d_{k j} t_{j}\right) \quad \text { and } \quad\left\{\beta_{k u} \prod_{\substack{j \in U \\ j \neq u}}\left(d_{k j} t_{j}\right) \mid u \in U\right\}
$$

is the unit ideal or of height 2. (If $U=\emptyset$, then $Z=Y$ by the construction. If $U=\{u\}$ and if $\beta_{k u}$ is a unit element, then this ideal is the unit. In other cases, this ideal is of height 2.) Therefore, the codimension of $Y \backslash Z$ in $Y$ is greater than or equal to two.

We can define the graded canonical module as in [5, Definition 3.1] using the theory of the equivariant twisted inverse functor (see [4]).

By Claim 3.5 above and $\left[5\right.$, Remark 3.2], we have $\omega_{T}=H^{0}\left(Y, \omega_{Y}\right)$. On the other hand, we have

$$
\begin{aligned}
\omega_{Y} & =\bigwedge^{s} \Omega_{Y / X^{\prime}} \otimes \pi^{*} \mathcal{O}_{X^{\prime}}\left(K_{X^{\prime}}\right) \\
& =\pi^{*} \mathcal{O}_{X^{\prime}}\left(\sum_{i} D_{i}^{\prime}\right)(-1, \ldots,-1) \otimes \mathcal{O}_{Y} \pi^{*} \mathcal{O}_{X^{\prime}}\left(K_{X^{\prime}}\right) \\
& =\pi^{*} \mathcal{O}_{X^{\prime}}\left(\sum_{i} D_{i}^{\prime}+K_{X^{\prime}}\right)(-1, \ldots,-1)
\end{aligned}
$$

where $(-1, \ldots,-1)$ denotes the shift of degree (see [4, Theorem 28.11]).

Then, we have

$$
H^{0}\left(Y, \omega_{Y}\right)=H^{0}\left(X^{\prime}, \pi_{*} \pi^{*} \mathcal{O}_{X^{\prime}}\left(\sum_{i} D_{i}^{\prime}+K_{X^{\prime}}\right)(-1, \ldots,-1)\right) .
$$

By the projection formula (see [4, Lemma 26.4]),

$$
\begin{aligned}
\pi_{*} & \pi^{*} \mathcal{O}_{X^{\prime}}\left(\sum_{i} D_{i}^{\prime}+K_{X^{\prime}}\right)(-1, \ldots,-1) \\
& =\left(\mathcal{O}_{X^{\prime}}\left(\sum_{i} D_{i}^{\prime}+K_{X^{\prime}}\right) \otimes \pi_{*} \mathcal{O}_{Y}\right)(-1, \ldots,-1) \\
& =\left(\mathcal{O}_{X^{\prime}}\left(\sum_{i} D_{i}^{\prime}+K_{X^{\prime}}\right) \otimes\left[\bigoplus_{\underline{n} \in W} \mathcal{O}_{X^{\prime}}\left(\sum_{i} n_{i} D_{i}^{\prime}\right)\right]\right)(-1, \ldots,-1)
\end{aligned}
$$




$$
\begin{aligned}
& =\left(\bigoplus_{\underline{n} \in W} \mathcal{O}_{X^{\prime}}\left(\sum_{i}\left(n_{i}+1\right) D_{i}^{\prime}+K_{X^{\prime}}\right)\right)(-1, \ldots,-1) \\
& =\bigoplus_{\underline{n} \in W+(1, \ldots, 1)} \mathcal{O}_{X^{\prime}}\left(\sum_{i} n_{i} D_{i}^{\prime}+K_{X^{\prime}}\right) .
\end{aligned}
$$

Therefore, we have

$$
\begin{aligned}
H^{0}\left(Y, \omega_{Y}\right) & =H^{0}\left(X^{\prime}, \bigoplus_{\underline{n} \in W+(1, \ldots, 1)} \mathcal{O}_{X^{\prime}}\left(\sum_{i} n_{i} D_{i}^{\prime}+K_{X^{\prime}}\right)\right) \\
& =\bigoplus_{\underline{n} \in W+(1, \ldots, 1)} H^{0}\left(X^{\prime}, \mathcal{O}_{X^{\prime}}\left(\sum_{i} n_{i} D_{i}^{\prime}+K_{X^{\prime}}\right)\right) \\
& =\bigoplus_{\underline{n} \in W+(1, \ldots, 1)} H^{0}\left(X, \mathcal{O}_{X}\left(\sum_{i} n_{i} D_{i}+K_{X}\right)\right) \\
& =M_{K_{X}} \cap t_{1} \cdots t_{s} k(X)\left[t_{1}, \ldots, t_{s},\left\{t_{j}^{-1} \mid j \notin U\right\}\right] .
\end{aligned}
$$

We have completed the proof of Theorem 1.3.

\section{REFERENCES}

[1] W. Bruns and J. Herzog, Cohen-Macaulay Rings, Cambridge Stud. Adv. Math. 39, Cambridge University Press, Cambridge, 1993. MR 1251956.

[2] E. J. Elizondo, K. Kurano, and K.-i. Watanabe, The total coordinate ring of a normal projective variety, J. Algebra 276 (2004), 625-637. MR 2058459. DOI 10.1016/j. jalgebra.2003.07.007.

[3] S. Goto, K. Nishida, and Y. Shimoda, The Gorensteinness of symbolic Rees algebras for space curves, J. Math. Soc. Japan 43 (1991), 465-481. MR 1111598. DOI 10. 2969/jmsj/04330465.

[4] M. Hashimoto, "Equivariant twisted inverses" in Foundations of Grothendieck Duality for Diagrams of Schemes, Lecture Notes in Math. 1960, Springer, Berlin, 2009, 261-478. MR 2490558. DOI 10.1007/978-3-540-85420-3.

[5] M. Hashimoto and K. Kurano, The canonical module of a Cox ring, Kyoto J. Math. 51 (2011), 855-874. MR 2854155. DOI 10.1215/21562261-1424884.

[6] Y. Hu and S. Keel, Mori dream spaces and GIT, Michigan Math. J. 48 (2000), 331348. MR 1786494. DOI 10.1307/mmj/1030132722.

[7] H. Matsumura, Commutative Ring Theory, Cambridge Stud. Adv. Math. 8, Cambridge University Press, Cambridge, 1986. MR 0879273.

[8] S. Mori, On affine cones associated with polarized varieties, Jpn. J. Math. (N.S.) 1 (1975), 301-309. MR 0439859.

[9] P. Samuel, Lectures on unique factorization domains, Tata Inst. Fund. Res. Stud. Math. 30, Tata Institute of Fundamental Research, Bombay, 1964. MR 0214579.

[10] Y. Shimoda, The class group of the Rees algebras over polynomial rings, Tokyo J. Math. 2 (1979), 129-132. MR 0541902. DOI 10.3836/tjm/1270473564. 
[11] A. Simis and N. V. Trung, The divisor class group of ordinary and symbolic blow-ups, Math. Z. 198 (1988), 479-491. MR 0950579. DOI 10.1007/BF01162869.

[12] K.-i. Watanabe, Some remarks concerning Demazure's construction of normal graded rings, Nagoya Math. J. 83 (1981), 203-211. MR 0632654.

Department of Mathematics

School of Science and Technology

Meiji University

Kawasaki 214-8571

Japan

kurano@isc.meiji.ac.jp 